\title{
Serological and molecular analysis of anti-Tja: Case report
}

\author{
Zhiyuan $\mathrm{Xu}^{1}$, Haochun Chang ${ }^{2,3,4}$, Xiaojie Zhu ${ }^{2,4}$, Chen $\mathrm{Cao}^{2,4}$, Tianhong Miao ${ }^{1}$, Jiwu Gong ${ }^{4,5}$, \\ Yu-Shiang $\operatorname{Lin}^{2,4^{*}}$ \\ ${ }^{1}$ Blood Group Lab, Beijing Red Cross Blood Center, Beijing 100088, china \\ ${ }^{2}$ Nobel Prize Winner Erwin Neher Research Institute, Jiangsu Zhongji Wantai Biological Pharmaceutical Co., Ltd. Jiangyin, Jiangsu 214400, china \\ ${ }^{3}$ Department of Clinical Medicine, School of Basic Medical Sciences, Peking University Health Science Center, Beijing 100191, china \\ ${ }^{4}$ The United Reference Laboratory for Blood Group Gene Detection, National Center for Clinical Laboratories, Beijing 100730, china \\ ${ }^{5}$ Department of Transfusion, National Center of Gerontology, Beijing Hospital, Beijing 100730, China
}

\begin{abstract}
$\mathrm{P}$ antigen frequency is very low in the Chinese population. However, the presence of anti-PlPP ${ }^{\mathrm{k}}$ (anti-Tja) is a huge risk for patients undergoing clinical transfusions and recurrent abortions. This report aims to describe $\mathrm{p}$ antigen and anti-Tja serological test features and suggests ways in which we may better identify the $\mathrm{p}$ antigen. Polymerase chain reaction was used to amplify A4GALT and B3GALNT1, which were then analysed for polymorphisms using Sanger sequencing. The A4GALT sequence results revealed c. 547-548delAT (HE818933), which resulted in a frame shift at aa 183 stopping at aa 281 (M183fs, 281X). Compared with the reference sequence, B3GALNTI did not show any variations in any of the subjects assessed. Eggs from Columba livia were used in the neutralised P substance test, but failed to neutralise anti-Tja. The serological test and molecular analysis confirmed that the P blood antigens are caused by A4GALTc. 547-548 AT deletion, and the neutralised P substance test cannot identify anti-PPIPk from RBC alloantibodies against high frequency antigens.
\end{abstract}

Keywords: anti-Tja, P blood group, p phenotype, A4GALT

\section{INTRODUCTION}

Very few reports have demonstrated the presence of anti-Tja in the Chinese population. According to recent news from the National Rare Blood Bank of China, type $\mathrm{P}$ is a very rare blood type, with only several cases found among millions of people ${ }^{[1]}$. Our report aims to describe features of a serological test for $\mathrm{p}$ antigen and anti-Tja, and suggests improved techniques for the identification of $\mathrm{p}$ blood antigens.

\section{CASE REPORT}

A 61-year-old female requiring exact diagnosis at

*Correspondence to: Yu-Shiang Lin, Nobel Prize Winner Erwin Neher Research Institute, Jiangsu Zhongji Wantai Biological Pharmaceutical Co., Ltd. Jiangyin, Jiangsu 214400, China. E-mail:1448639963@qq.om Conflict of interests: The authors declared no conflict of interests. our centre due to antibody identification demonstrated suspect to alloantibodies against high frequency antigens of red blood cells. The antibody screening and identification tests showed that all cells had an agglutinin level of $3+$ when combined with patient plasma. Meanwhile, the auto-control and direct Coombs test were negative. The phenotype was reported as Plnegative, $\mathrm{Fy}(\mathrm{a}+\mathrm{b}-)$ and $\mathrm{Jk}(\mathrm{a}-\mathrm{b}+)$.

\section{Samples and serological tests}

EDTA-treated, anti-coagulated peripheral blood was stored at $4{ }^{\circ} \mathrm{C}$. Serological tests and DNA extraction were performed within 12 hours of blood acquisition. The DNA was purified from buffy coat samples using micromagnetic technology with a commercial kit (Magnetic Bead, Texas Biotechnology Co., Ltd., Xiamen, China) and an automatic instrument (EZ 
Bead-32, Texas Biotechnology Co., Ltd., Xiamen, China.). All DNA samples were stored at $-40^{\circ} \mathrm{C}$ until molecular analysis.

Routine $\mathrm{ABO}, \mathrm{RhD}$ blood typing and antibody screening and identification used micro-column tests [ABO RhD Blood Typing Card, ABO cells, screen cells, and Anti-Human Globulin Card (anti-IgG and anti-C3d), Jiangsu Libio Biotech Co.Ltd, Jiangsu, China; Panel cells, Jiangsu ZhongJi Wantai Biological Pharmaceutical Co Ltd, Jiangsu, China]. P1 phenotyping was done using monoclonal anti-Pl (Jiangsu ZhongJi Wantai Biological Pharmaceutical Co Ltd, Jiangsu, China).

\section{The neutralised P1 substance test}

Turtle dove eggs were used for the neutralised Pl substance test. The egg was diluted $100 \times$ through normal saline and mixed up and down 20 times. This diluted solution $(10 \mu \mathrm{L})$ was mixed with $200 \mu \mathrm{L}$ of patient plasma and incubated at room temperature for $15 \mathrm{~min}$. Monoclonal anti-Pl was used as a positive control and normal saline as a negative control. After incubation, the neutralised plasma reacted with the $\mathrm{Pl}+$ screen cells. The neutralised P substance test was negative. When the monoclonal anti-Pl and saline controls were incubated with $\mathrm{P}$ substance and crossmatched with $\mathrm{Pl}+$ cell, the result was negative, in contrast with when plasma from a patient with $\mathrm{p}$ phenotype was used, which resulted in a recording of $3+$.

\section{Fluo-genotyping}

Multi-erythrocytic blood group genotyping was performed by polymerase chain reaction (PCR) with sequence-specific primers using a commercial kit (Human Multi-Erythrocytic Antigen Fluo-Genotype; Jiangsu ZhongJi WanTai Biological Pharmaceutical Co., Ltd., Jiangsu, China). The fluo-genotype was Ccee for RHCE, Lw $(a+b-)$ for Landsteiner-Wiener, Fy(a+b-) for Duffy, Jk(a-b+) for Kidd, MMssMur- for MNSs, Di(a-b+), Wr(a-b+) for Diego kk and Kp(a-b+) for kell, Co(a+b-) for Colton, Do(a-b+) for Domocrock, $\mathrm{Au}(\mathrm{a}-\mathrm{b}+)$ for Augestine, and $\mathrm{Yt}(\mathrm{a}+\mathrm{b}-)$ for $\mathrm{Yt}$ (Fig 1). These results excluded related common RBC alloantibodies against high frequency antigens, including the Jknull, RH variant, Fya-, Dib-, Lw a-, Sc+, Colton and Domcrock, among others.

\section{Molecular analysis}

Sanger sequencing of the A4GALT and B3GALNT1 genes was performed using a commercial Rare Blood Group Sequencing Kit [P blood group (A4GALT and B3GALNT1) sequence kit, Jiangsu ZhongJi WanTai Biological Pharmaceutical Co., Ltd, Jiangsu, China].
Sequencing of PCR-purified products was performed by GENEWIZ (Jiangsu, China) and the results were analysed using sequence analysis software (GeneiousR9; Auckland, New Zealand). The A4GALT (GenBank No. AJ245581) and B3GALNT1 (GenBank No. AB050855) allele sequence templates were used as references for analyses and to mark mutations. JMH, Vel, Lan, Lutheran and p. A4GALT variants were confirmed while B3GALNT1 showed the same sequence as the reference. A4GALT sequencing revealed a c. 547-548delAT (HE818933) variant (Fig 2), which results in a frame shift at aa 183 stopping at aa 281 (Ml83fs, 281X). When compared to the reference, the B3GALNT1 sequence was invariant for all subjects.

\section{DISCUSSION}

Few studies have examined the $\mathrm{P}$ blood group variant in Chinese people. Two variants of A4GALT have been described, and only one study has reported that anti-Tja induces recurrent abortion ${ }^{[2-5]}$. However, to our knowledge, at least four cases of RBC alloantibodies against high frequency antigens are strongly suspected to be due to anti-Tja. To date, there is no serological test for the rapid identification of antiTja. Our study used the neutralised P substance test and molecular tests to examine p phenotype, and our results have demonstrated that sequence analysis is a superior method to examine $\mathrm{P}$ blood groups, especially for $\mathrm{p}$ phenotype.

Expansion of the P system was described in 1955 by the Sanger group, following the injection of rabbits with human red cells ${ }^{[6]}$. In the "Practical Guide to Transfusion Medicine" by Marian Petrides et al., there is a chapter on "P Blood Group and GLOB Collection" by Laura Cooling. This indicated that two glycotransferases synthesised from the common precursor, lactosylceramide, form "P1", "Pk","P" or "LKE" antigens on the red blood cell membrane through the globoside and paragloboside series, including $\alpha$ 1,4- galactosyltransferase (A4GALT), which is located at chromosome $22 \mathrm{q} 13.2$, and $\beta 1,3-\mathrm{N}$ acetylgalactosaminyltransferase (B3GALNT1), which is located at chromosome $3 \mathrm{q} 25+^{[2,6]}$. In the globoside series pathway, A4GALT catalyses the transfer of galactose to lactosylceramide ( $\mathrm{Gb} 2$ ) to produce $\mathrm{Gb} 3$ (Pk antigen) and B3GALNT1 catalyses the transfer of acetylgalactosamine to $\mathrm{Gb} 3$ to produce $\mathrm{Gb} 4$ (P antigen $)^{[7-8]}$. In contrast, in the paragloboside series pathway, ceramide first links to Gb2 and A4GALT, then transfers galactose to produce $\mathrm{Pl}$ antigen. Alloantibodies against the $\mathrm{P}$ system are more commonly of the Pl phenotype, including anti-Pl, due to weak 


\begin{tabular}{|c|c|c|l|c|l|c|l|}
\hline \multicolumn{2}{|c|}{ ID: Sample 03 } & RH & Ccee & LW & LW(a+b-) & Duffy & Fy $(a+b-)$ \\
\hline Kidd & Jk(a-b+) & MNSs & MMss & Mur & Mur- & Sciana & Sc- \\
\hline Diego & Di $(a-b+)$ & Wright & Wr $(a-b+)$ & Kell & kk & Kp & Kp(a-b+) \\
\hline Co & Co $(a+b-)$ & Do & Dor $((a-b+)$ & Augestine & Au((a-b+) & Yt & Yt(a+b-) \\
\hline
\end{tabular}

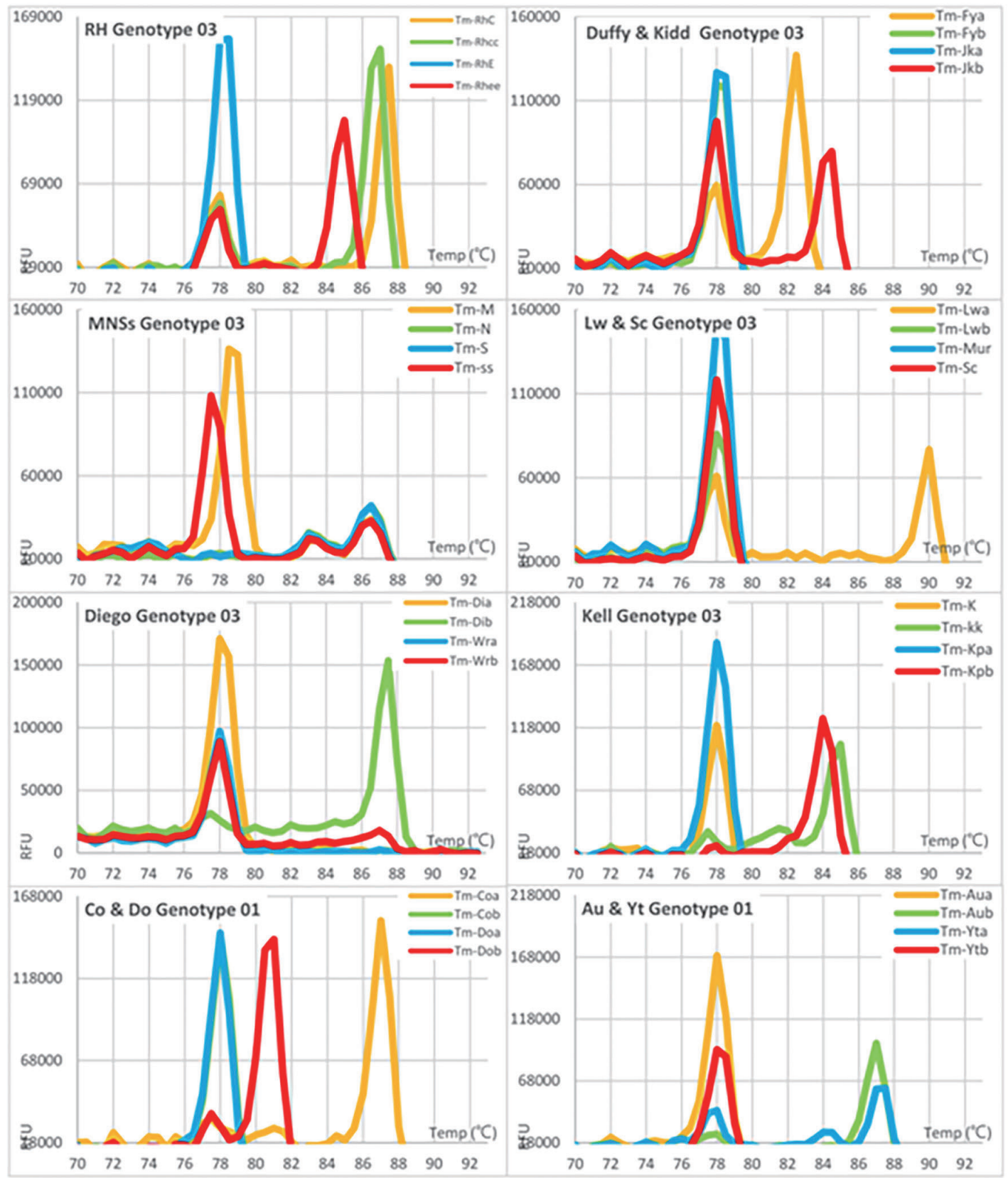

Fig. 1 The fluo-genotyping of multi-erythrocytic blood groups for the patient. $\mathrm{X}$ axis means temperature and $\mathrm{Y}$ axis means $\log$ value of Fluorescence. The curve means internal control for PCR at $76^{\circ} \mathrm{C}-78^{\circ} \mathrm{C}$. The curve presented shows positive for PCR through sequence specific primers over $80^{\circ} \mathrm{C}$. With the exception of PCR for MNSs sequence specific primers, the curve mean internal control for PCR at over $80^{\circ} \mathrm{C}$, while the curve presented means positive for PCR through sequence specific primers between $76^{\circ} \mathrm{C}-78^{\circ} \mathrm{C}$.

A4GALT-formed $\mathrm{P}^{\mathrm{k}}$ and $\mathrm{P}$ antigen.The $\mathrm{P} 2$ phenotype has a lower frequency and consists of anti-P alloantibodies due to the B3GALNT1 variant resulting in a lack of $\mathrm{P}$ antige ${ }^{[9]}$. Very rare alloantibodies against the P system include anti-PPIP ${ }^{k}$ (also called anti-Tja),

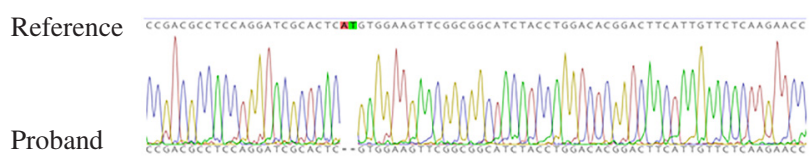

Fig.2 The A4GALT variat of c. 547-548 del AT (HE818933) 
which results from A4GALT variants ${ }^{[10]}$.

We attempted to use Columba livia eggs as a neutralised $\mathrm{P}$ substance with anti-P1PP ${ }^{\mathrm{k}}$ from a $\mathrm{p}$ phenotype with A4GALT 547-548 homozygous deletions. These neutralization tests were unsuccessful. It has been reported that gastric carcinoma produces 'illegitimate' $\mathrm{P}$ antigens, and that these $\mathrm{P}$ antigens act as receptors for the Parvo B 19 virus and HIV-1. It is almost certain that anti-PPIP ${ }^{k}$ in the sera of $p$ women is the cause of the observed abortions and mild haemolytic diseases in new-borns. One reported case known to us involved a woman from the Yunnan province in China, which is abundant in minorities, from where there is an increased incidence of anti-Tja. Thus, the conduction of a localized systemic investigation would likely yield positive results.

In summary, the serological tests combined with molecular analyses was able to successfully identify the $\mathrm{p}$ blood antigens caused by A4GALTc. 547-548 deletion AT variant and the neutralised $\mathrm{P}$ substance test cannot identify anti-Tja from RBC alloantibodies against high frequency antigens.

\section{References}

[1] National Rare Blood Bank of China Supplied Type $p$ blood to Japanese Patient[2]. https://www.chinarareblood.cn/news-77.aspx.

[2] Li X, Diao X, Xia X, et al. A novel mutation in A4GALT was identified in a Chinese individual with $p$ phenotype[J]. Transfusion, 2017, 57(1):215-216.
[3] Xu X, Hong X, Ma K, et al. A rare p phenotype caused by a 26-bp deletion in alpha 1,4-galactosyltransferase gene[J]. Zhonghua Yi Xue Yi Chuan Xue Za Zhi(in Chinese), 2013 30(3):309-12.

[4] Chu C, Shen WM, Chen H, et al. Rare blood type of Jk(a-b-), Di b-、Wr b-、K+、Ena-、Tja-、Ge in Shanghai[J]. China Journal of Blood Transfusion(in Chinese), 2002, 15(4):232-233.

[5] Chen A, Zhang L. A case report of anti-Tja induced Habitual abortion [J]. Laboratory Medicine, 2004, 19(3):276(in Chinese).

[6] Landsteiner K, Levine P. On individual differences in human blood[J]. Proc Soc Exp Biol NY,1927, 24:941942 .

[7] Francois-Gerard CJ, Fau-Andre JA, Fau-Gerday AC, et al. Demonstration of the existence of a specific blood-group $\mathrm{Pl}$ antigenic determinant in turtle-dove ovomucoid[J]. Transfus Immuno-H é mat, 1980, 23:579-588.

[8] Khoo KH, Nieto A, Morris HR,et al. Structural characterization of the N-glycans from Echinococcus granulosus hydatid cyst membrane and protoscoleces[J]. Mol Biochem Parasitol, 1997, 86(2): 237 -248.

[9] Fau-Kaarsalo AE, Fau-Melartin EL, Fau-Tippett LP, et al. The red cell antigen $\mathrm{Pk}$ and its relationship to the $\mathrm{P}$ system:the evidence of three more $\mathrm{Pk}$ families[J].Vox Sang, 1965, 10(4):385-404.

[10] Levine P, Bobbitt OB, Waller RK, et al. Isoimmunization by a new blood factor in tumor cells[J]. Proc Soc Exp Biol Med, 1951, 77(3):403-405.

(Received 22 April 2018, Revised 02 June 2018, Accepted 05 June 2018) 